\title{
A NOTE ON FRACTURE TOUGHNESSES OF UNIDIRECTIONAL COMPOSITES
}

\section{S. Y. Zhang}

Institute of Mechanics, Chinese Academy of Sciences

Beijing 100080, People's Republic of China

In-plane fracture behaviour of unidirectional fibre composite materials has been investigated by many researchers. Ashby et al. [1] and Maiti [2] analysed the in-plane fracture toughnesses of the two cases, $i_{0} e_{0}$, the crack lying parallel to the fibres and crack normal to the fibres. Many experimental results showed that the fracture toughness of the case of the crack normal to the fibres, $\mathrm{K}_{\mathrm{Ic}}^{\mathrm{n}}$ is much larger than that of crack parallel to the fibres, $\mathrm{K}_{\mathrm{Ic}}^{\mathrm{a}}$. Ashby et $\mathrm{lc}$ al. derived the relationship between the two toughnesses by using stress approach. This relationship is as follows:

$$
\frac{\mathrm{K}_{\mathrm{Ic}}^{\mathrm{n}}}{\mathrm{K}_{\mathrm{Ic}}^{\mathrm{a}}}=\frac{\sqrt{2}\left(\mathrm{D}^{\frac{1}{4}}+\mathrm{C}^{\frac{1}{4}}\right)}{\sqrt{\mathrm{CD}}}
$$

where

$$
\begin{aligned}
& \mathrm{D} \\
& \mathrm{D}
\end{aligned}=\frac{1}{2}\left(\frac{\mathrm{E}_{2}}{\mathrm{G}_{12}}-2 v_{21}\right) \mp \frac{1}{2}\left[\left(\frac{\mathrm{E}_{2}}{\mathrm{G}_{12}}-2 v_{21}\right)^{2}-\frac{\mathrm{E}_{2}}{\mathrm{E}_{1}}\right]^{\frac{1}{2}}
$$

In deriving (1), Ashby et al. assumed that at the onset of Eracture, the stress fields of the two configurations (i.e., crack parallel to and normal to the fibres) are identical. So, for the case of crack normal to the fibres, the onset of fracture is assumed to be caused by normal stress only, whereas the effect of shear stress on fracture is ignored.

In reality, there is no ground in the assumption. In the present research note, a brief account on this problem is presented.

For the case of crack parallel to the fibres, the fracture is of mode $I$, the normal stress causing the onset of fracture can be expressed as (Fig. 1 and Fig. 2):

$$
\sigma_{y y}^{a}=\frac{x_{I}^{a}}{\sqrt{2 \pi r}}
$$

According to the point-stress fracture criterion [3], when the stress value at the point being $r$ ahead from the crack tip equals the strength of the material, the fracture will occur.

$$
\sigma_{y y}^{a}=\frac{K_{I}^{a}}{\sqrt{2 \pi r_{0}}}=\mathrm{T}
$$

where $r$ is the characteristic dimension of the point-stress fracture criterion。 ${ }^{\circ} \mathrm{T}$ is the transverse tensile strength of the material. Here, the effect of the stress component parallel to the fibres on fracture is ignored. 
For the case of crack normal to the fibres, the fracture is of mixed mode. The contribution of the shear stress, $\tau^{\mathrm{h}}$ to the fracture can not be ignored. Following the notations of [1] and $x y$ [2], the stress components can be given as:

$$
\begin{aligned}
& \sigma_{x x}^{n}=\frac{k_{I}^{n}}{\sqrt{2 \pi r}} \operatorname{Re}\left\{\frac{\mu_{1}^{n} \mu_{2}^{n}}{\mu_{1}^{n}-\mu_{2}^{n}}\left(\sqrt{\mu_{2}}-\sqrt{\mu_{1}^{n}}\right)\right\} \\
& \sigma_{y y}^{n}=\frac{k_{I}^{n}}{\sqrt{2 \pi r}} \operatorname{Re}\left\{\frac{1}{\mu_{1}^{n}-\mu_{2}^{n}}\left(\frac{\mu_{1}^{n}}{\sqrt{\mu_{2}^{2}}}-\frac{\mu_{2}^{n}}{\sqrt{\mu_{1}^{n}}}\right)\right\} \\
& \tau_{x y}^{n}=\frac{K_{I}^{n}}{\sqrt{2 \pi 4}} \operatorname{Re}\left\{\frac{\mu_{1}^{n} \mu_{2}^{n}}{\mu_{1}^{n}-\mu_{2}^{n}}\left(\frac{1}{\sqrt{\mu_{1}^{n}}}-\frac{1}{\sqrt{\mu_{2}^{n}}}\right)\right\}
\end{aligned}
$$

where

$$
\left.\begin{array}{c}
\left(\mu_{1}^{n}\right)^{2} \\
\left(\mu_{2}^{n}\right)^{2}
\end{array}\right\}=\frac{1}{2}\left(\frac{E_{2}}{G_{12}}-2 v_{21}\right)+\left[\left(\frac{E_{2}}{G_{12}}-2 v_{21}\right)^{2}-4 \frac{E_{2}}{E_{1}}\right]^{\frac{1}{2}}
$$

Where $E_{1}, E_{2}, G_{12}$, and $v_{21}$ are elastic constants of the material; direction1 is the 12 fibre 21 direction.

Mixed mode fracture should be characterised by failure criterion. Omitting the effect of the stress component parallel to the fibres on fracture (as for the case of crack parallel to the fibres), the Tsai-Hill criterion is reduced to

$$
\left(\frac{\sigma_{x x}^{n}}{T}\right)^{2}+\left(\frac{T_{x y}^{n}}{S}\right)^{2}=1
$$

where $\mathrm{S}$ is shear strength of the material. The relationship between $\mathrm{K}_{\mathrm{Ic}}^{\mathrm{a}}$ and $\mathrm{K}_{\mathrm{Ic}}^{\mathrm{n}}$ can be obtained from the following:

$$
\left(\frac{\sigma_{x x}^{n}}{T}\right)^{2}+\left(\frac{\tau_{x y}^{n}}{s}\right)^{2}=\left(\frac{\sigma_{y y}^{a}}{T}\right)^{2}
$$

Assuming that the characteristic dimension $r$ of the case of crack normal to the fibres is identical to that of crack parallel to the fibres, the final relationship between the two in-plane fracture toughnesses $\mathrm{K}_{\mathrm{Ic}}$ and $\mathrm{K}_{\mathrm{Ic}}^{\mathrm{n}}$ is obtained.

$$
\frac{\mathrm{K}_{\mathrm{Ic}}^{\mathrm{n}}}{\mathrm{K}_{\mathrm{Ic}}^{\mathrm{a}}}=\left\{\left[\frac{\sqrt{\mathrm{CD}}}{\sqrt{2}\left(\mathrm{C}^{\frac{1}{4}}+\mathrm{D}^{\frac{1}{4}}\right)}\right]^{2}+\left[\left(\frac{\mathrm{T}}{\mathrm{S}}\right) \frac{(\mathrm{D} C)^{\frac{1}{4}}}{\sqrt{2}\left(\mathrm{D}^{\frac{1}{4}}+\mathrm{C}^{\frac{1}{4}}\right)}\right]^{2}\right\}^{-\frac{1}{2}}
$$

when $\mathrm{T} / \mathrm{S}=0,(11)$ reduces to $(1)$ 。 
Two sets of experimental data quoted in [2] were calculated by using (1). The results are given in Table 1 and compared with the calculated results of (1) and the experimental data。 For most commonly used epoxy-based fibre composites, the value of the ratio $\mathrm{T} / \mathrm{S}$ approximately equals to $\frac{1}{2} \sim 1$. It can be seen that the results of (11) are much closer to the experimental data than those of (1)。

\section{REFERE ICES}

[1] M.F. Ashby, K.E. Easterling, R. Harrysson, and S.K. Maiti, Proceedings of Royal Society (London) A398 (1985) 261-280.

[2] S.K. Maiti, International Joumal of Fracture 32 (1986) R29-R32.

[3] J.M. Whitney and R.J. Nuismer, Joumal of Composite Materials 8 (1984) 253-265.

7 July 1987

Table 1. Comparison of the calculated results with experimental data

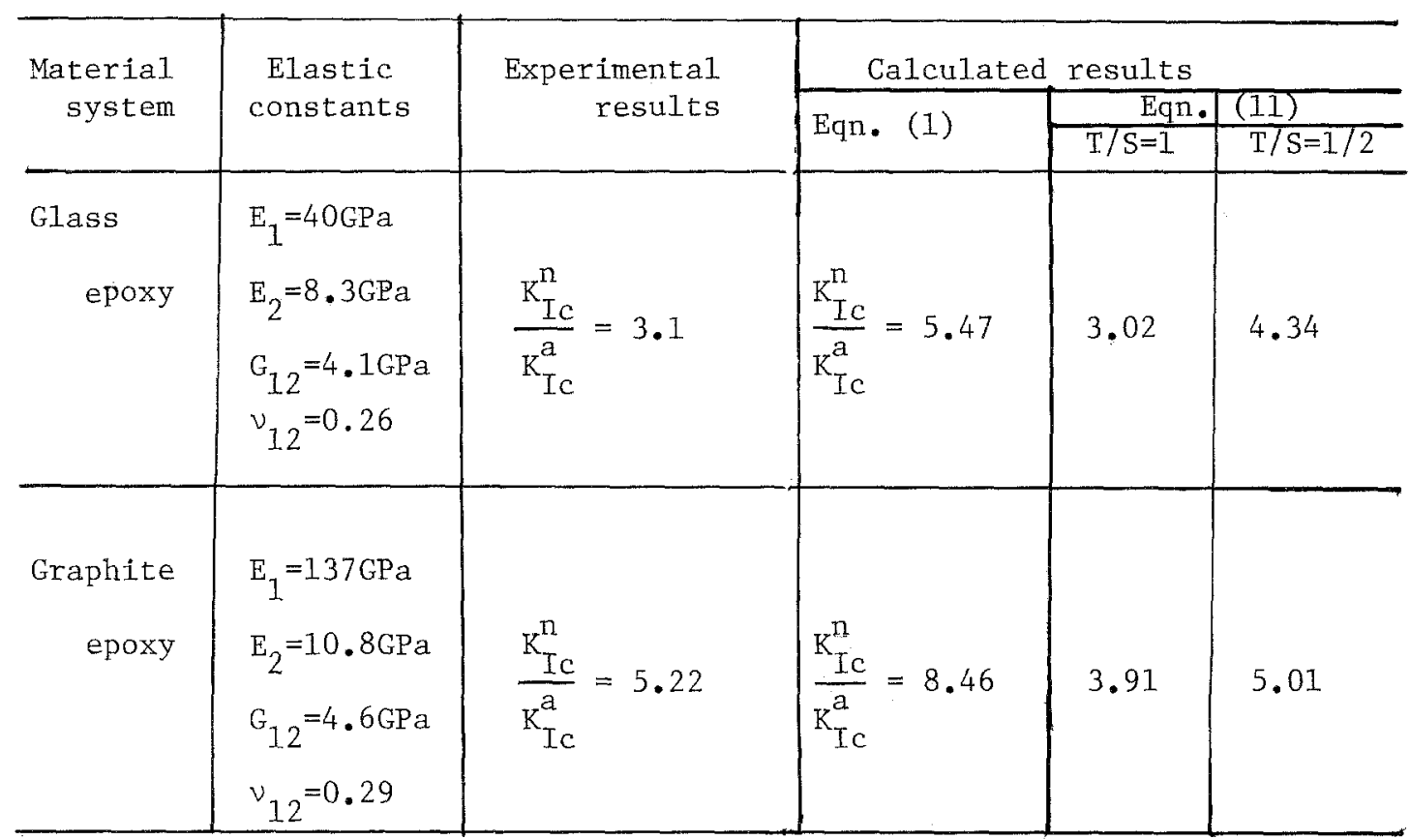




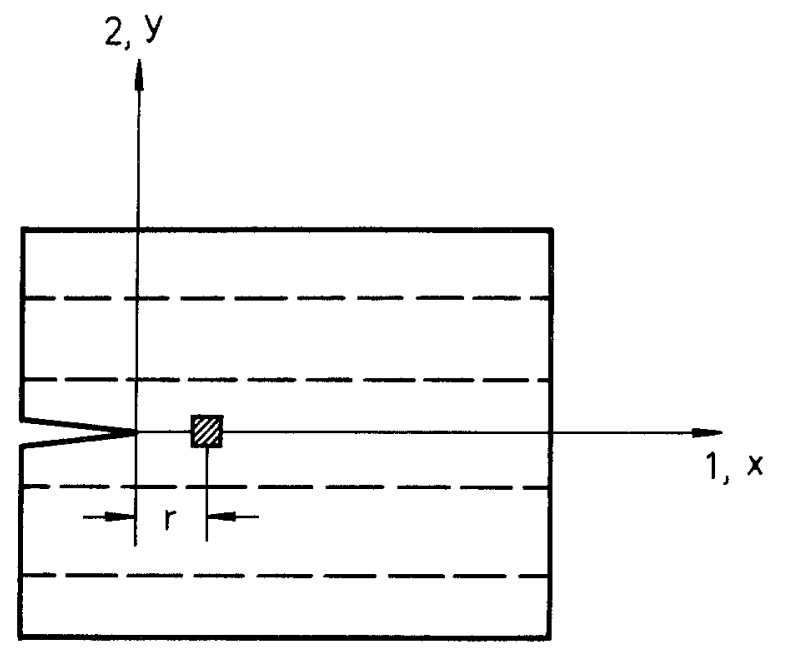

Figure 1. Crack parallel to fibres.

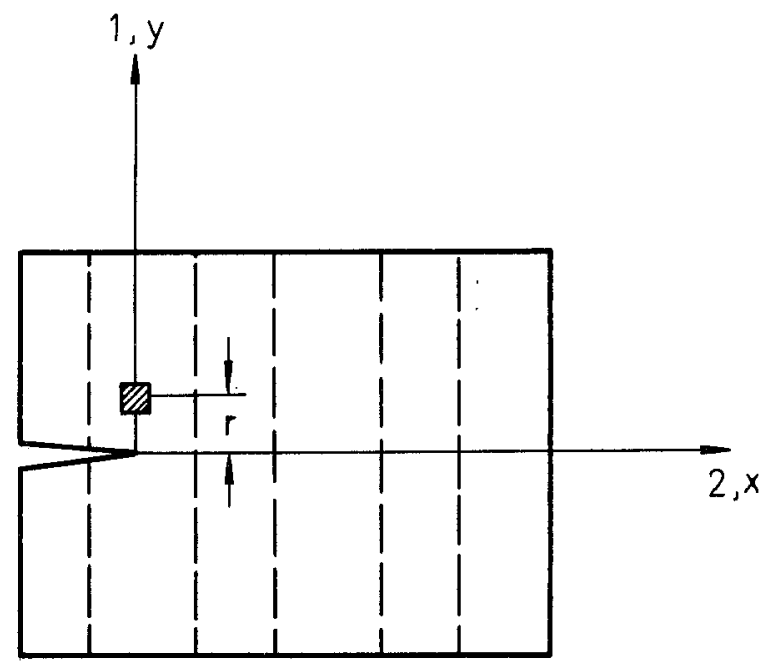

Figure 2. Crack normal to the fibres. 